\title{
Context-Dependent Enhancements for Radiological Images
}

\author{
B. Plessis, M. Goldberg, R. Dillon, J. Tombaugh, J. Robertson, G. Bélanger, and N. Hickey
}

\begin{abstract}
Many techniques have been proposed to enhance radiographic images and each of them may be optimal depending upon the circumstances. However, the problem confronting the radiologist or the physician is which enhancement to use and how to select the parameters when a specific feature is to be emphasized. At the University of Ottawa, our research work is oriented towards automatic contextdependent enhancements. Our approach attempts to match the three phases involved in viewing a radiograph: getting a global impression, analyzing the objects and the local features, and focusing on the image perturbations. In this article, we report on enhancements to support the first two phases in the case of chest radiographs and on the applicability of gray level reversal transformations.

(C) 1989 by W.B. Saunders Company.
\end{abstract}

C HEST RADIOGRAPHS are the most important single images in radiology. ${ }^{1}$ With the advent of digital-based workstations, there is a growing interest in digitizing standard chest radiographs. Two main physical parameters that are important in evaluating the quality of a digitized chest radiograph are spatial resolution and contrast resolution. Spatial resolution is related to the ability to discriminate fine detail in the field of view. Contrast resolution refers to the smallest noticeable difference in luminance between a small object and its background. The jury is still out on the minimum spatial resolution required for diagnostic quality. The results given in literature range from 1,000 to 4,000 lines for a standard chest radiograph with 10 to 12 bits for contrast. An important practical consideration is the cost and availability of technology, which at

From the Telecommunications Research Institute of Ontario, University of Ottawa Medical Communication Research Centre; Department of Psychology, Carleton University; and Department of Radiological Sciences, Ottawa Civic Hospital, Canada.

Supported by the Telecommunications Research Institute of Ontario (TRIO), Bell Canada, and Bell-Northern Research (BNR). Brigitte Plessis is supported by a grant from the External Affairs Department of the Canadian Government (administrated by WUSC).

Address reprint requests to B. Plessis, $M S c A$, Telecommunications Research Institute of Ontario, University of Ottawa Medical Communication Research Centre, 161 Louis Pasteur, Ottawa, Ontario KIN 6N5, Canada.

(1) 1989 by W.B. Saunders Company.

0897-1889/89/0202-0010\$03.00/0 present implies the use of displays with 1,000 lines of resolution and eight bits of contrast.

Image processing for visual enhancement has been the subject of extensive research in many domains including medical imagery. ${ }^{2}$ We can distinguish three types of enhancements to apply to digitized chest radiographs: global, global adaptive, and local enhancements. Global enhancements perform the same process to each pixel in the image and are independent of the spatial coordinates; examples include histogram equalization, gray-scale transformations, and edge enhancement. ${ }^{3}$ This independence of spatial coordinates means that they are very easily implemented. However, this also implies that they do not not adapt to the local context on the image, and the results reported have proven to be disappointing. ${ }^{4}$ Global adaptive enhancement techniques are applied to the entire image but vary depending on the context. For example, in adaptive histogram equalization ${ }^{5}$ the local contrast is increased by remapping the gray-level values in some window to equalize the local histogram. A variation of this histogram equalization technique ${ }^{6}$ alters the contrast in the mediastinum and subdiaphragmatic areas while keeping the lung unchanged. Adaptive filtration' is another example of global adaptive technique. It uses the histogram of the image to determine the regions in which edge enhancement is applied.

Local enhancements are performed by processing only a portion of the image, leaving the rest invariant. $^{3}$ An application would be used for highlighting specific features. Any of the enhancement techniques applied on a global level could also be applied on a local level. Local enhancements can be considered an alternative to adaptive or context-dependent enhancements.

As image enhancement involves the improvement of visual quality, some form of subject evaluation is often necessary. A number of results have been reported on clinical experiments in which radiologists are asked to compare conventional analog and digitized chest images that have undergone various enhancements. ${ }^{8,9}$ Other trials used enhancements to highlight specific pathologies. ${ }^{10-12}$ 
One further consideration is the amount and degree of user control required in the imageenhancement process. The end goal would be a system, such as that proposed by Blume and Kamiya, ${ }^{13}$ which claims to automatically provide on the screen a satisfactory image whatever its contextual characteristics. It consists of semiautomatically scaling the clinically useful data span to the available data range. A detector auto-ranging method is used to renormalize the image that has been previously filtered with a nonlinear unsharp mask technique.

Our research work is oriented towards automatic context-dependent enhancements. In viewing a radiograph, there are three phases. ${ }^{14}$ The first phase corresponds to the "gestalt" or to getting a global impression. The second phase consists of analyzing the objects and the local features (eg, size and borders of the heart and lung vessels). The third phase refers to focal attention and scrutiny of perturbations (eg, shape and density of small nodules). To support these three different phases, three different types of enhancement procedures are proposed. The role of the first is to yield an appropriate compromise or baseline image matched to the "gestalt" requirement. The second enhancement is then invoked to aid in the analysis of local features. Finally, various local enhancements are then called to highlight the areas of interest.

In this article, we focus on the first two enhancement phases as applied to digitized chest radiographs. For the first phase, we report results on user evaluation trials of different enhancements that are applied to the original digitized images to yield baseline images. For the second phase, we report on the performance of simple global adaptive gray-scale manipulations to support local feature analysis, specifically for the lung, heart, and spine. In addition, we report results of user trials on the applicability of various gray-level reversal transformations.

\section{MATERIALS AND METHODS}

A laser scanner manufactured by Konica (Konishiroka, Burlingame, CA) is used to digitize the radiographs. For standard $35 \mathrm{~cm} \times 43 \mathrm{~cm}$ chest radiographs, it provides images of size $2,000 \times 2,430$ pixels with 1,024 gray levels. The corresponding pixel size is $175 \mu \mathrm{m} \times 177 \mu \mathrm{m}$. The scanner also offers the capability of selecting one of four optical density range settings: $(0-4),(0-2),(1-3),(2-4)$. For display requirements, the digitized images are low-pass filtered and sub-sampled to $1,000 \times 1,200$ pixels and the number of gray levels per pixel is also reduced from 1,024 to 256. The images are displayed on a black-and-white Philips monitor at a horizontal scan rate of $84 \mathrm{kHz}$ with a $104 \mathrm{MHz}$ band width. This monitor is operated on a portrait style to preserve the aspect ratio of chest radiographs. An IP-2K Imagraph image memory board of 2 Mbytes is used to refresh the screen at a rate of $60 \mathrm{~Hz}$. All these components are controlled by a Compaq Deskpro 386 , running at $20 \mathrm{MHz}$, with 13 Mbytes of internal RAM and 130 Mbytes of hard disk memory. The experiments were performed in collaboration with the Department of Radiological Sciences at the Ottawa Civic Hospital, a 950-bed tertiary care teaching hospital affiliated with the University of Ottawa.

The chest images were selected from three different departments of the hospital where different acquisition techniques are used. In the Emergency Department, images are exposed at $90 \mathrm{kVp}$. The same amount of energy is used in the Intensive Care Unit but the images have disproportionate shapes as patient positions are difficult to control. Images from these two departments were digitized with a reading setting of (0-2). In the Radiology Department, images are exposed at $1.45 \mathrm{kVp}$ and an anatomically shaped filter is used to reduce the amount of energy going through the lung. As a result, the lung area, mediastinum, and subdiaphragmatic areas are equally contrasted. These radiographs were digitized with a reading setting of (0-4). Two posterior-anterior images were obtained from each department and constitute the test set.

A digitized image can be defined as a two-dimensional array of integers $f(i, j)$ where $f(i, j)$ represents the intensity of the pixel $(i, j)$. Pixel intensities vary in a range defined by the possible gray-levels span. An enhancement technique is a remapping of all or part of the pixel intensities of the input image to new values through a mathematical transformation $T$ and yields a new image $g(i, j)$. If $I$ defines the entire set of image spatial coordinates, then the three types of enhancement can be expressed as follows:

Global enhancement: $g(i, j)=T[f(i, j)]$;

Adaptive enhancement: $g(i, j)=T_{i, j}[f(i, j)]$, where $T_{i, j}$ depends on the local statistics of the image; and

Local enhancement:

$$
\begin{array}{ll}
g(i, j)=T_{D}[f(i, j), & (i, j) \in D \subset I \\
g(i, j)=f(i, j), & (i, j) \in I-D
\end{array}
$$

where $D$ is a subset of $I$.

\section{Experiment 1: Optimization of the First Overview Image}

The objective of this first experiment is to find the best baseline image. By baseline image, we mean the first image displayed on the screen and read by the user during the "gestalt" phase. In particular, we want to answer the following questions: What is the enhancement that yields the best baseline image? Do the preferences vary between radiologists and other clinicians? Do the preferences depend on the radiograph acquisition technique? How do the preferred baseline images compare with the analog films? We are only interested in the physician's first impression of the displayed image, assuming that he does not know anything about the analog film or about the patient's history.

Although our images are digitized to 1,024 contrast levels, 
corresponding to $10 \mathrm{bits}$, our work stations only offer a range from 0 to 255 . The baseline images are obtained by transforming the digitized inages having 1,024 gray levels to images having 256 gray levels. All six types of transformations ${ }^{3,4,15}$ are tested with $A, B, C$, and $D$ as the normalization factors:

1. Simple truncation, $((233 / 1023) . x)$, where $I n t$ is the integral part of $x$

2. Exponential and power functions that expand the higher end of the scale, $T(x)=$ Int $(A \cdot \exp (x))$ or $T(x)=\operatorname{Int}\left(B x^{n}\right)$

3. Logarithmic and $n$th root functions that expand the lower end of the scale, $T(x)=\operatorname{Int}(C \cdot \log (x))$ or $T(x)=$ Int $\left(D x^{1 / n}\right)$

4. Piecewise linear functions that expand either the first or the last part of the scale,

$$
T(x)=\left\{\begin{array}{l}
\operatorname{Int}(A, x), x \in[0, M] \\
\operatorname{Int}(B, x+C), x \in[M, 1023]
\end{array}\right.
$$

5. Polynomial functions and piecewise nonlinear functions that expand the middle part of the scale,

$$
\begin{gathered}
T(x)=\operatorname{Int}\left(\mathrm{Ax}^{3}+\mathbf{B x ^ { 2 }}+\mathbf{C x}+\mathbf{D}\right) \\
\text { or } \\
T(x)=\left\{\begin{array}{l}
\operatorname{Int}(\mathrm{T} 1(\mathrm{x})), \mathrm{x} \in[0, \mathrm{M}] \\
\operatorname{Int}(\mathrm{T} 2(\mathrm{x})), \mathrm{x} \in[\mathrm{M}, 1023]
\end{array}\right.
\end{gathered}
$$

6. Global histogram equalization that equalizes the gray level distribution and stretch the contrast in low-contrasted areas, $T(x)=\operatorname{Int}(\operatorname{A.Cum}(\mathrm{x})) ; \operatorname{Cum}(x)$ is the cumulative distribution function.

We now provide additional information about the particular form of transformation used and its intended effect. We note that transformation 1 (Fig 1) has no enhancement effect. For both transformations 2 and $3, n$ is set equal to 2 . The effect of transformation 2 is to enhance the bright areas and contrast the dark areas (Fig 2). This effectively enhances the heart and spine and de-emphasizes the lung field. These effects are reversed in the case of transformation 3 (Fig 3). For transformation 4, we chose to expand the lower end of the

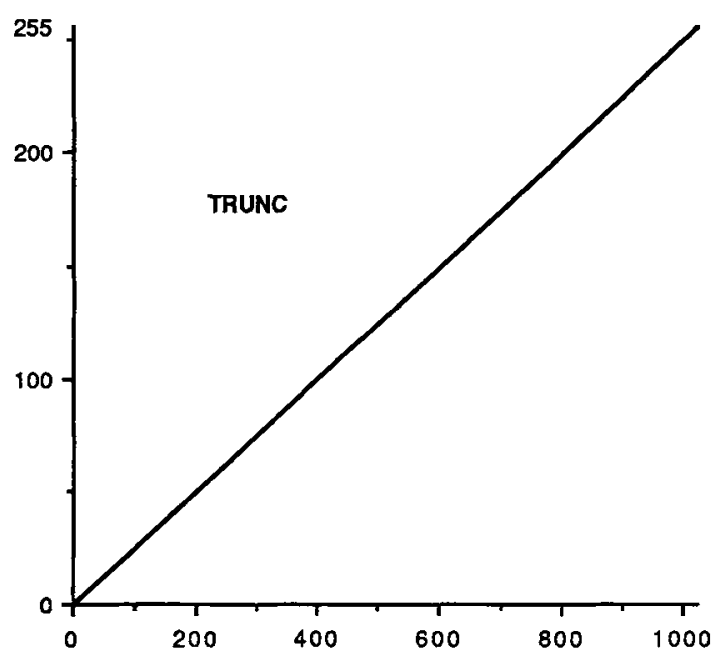

Fig 1. Simple truncation.

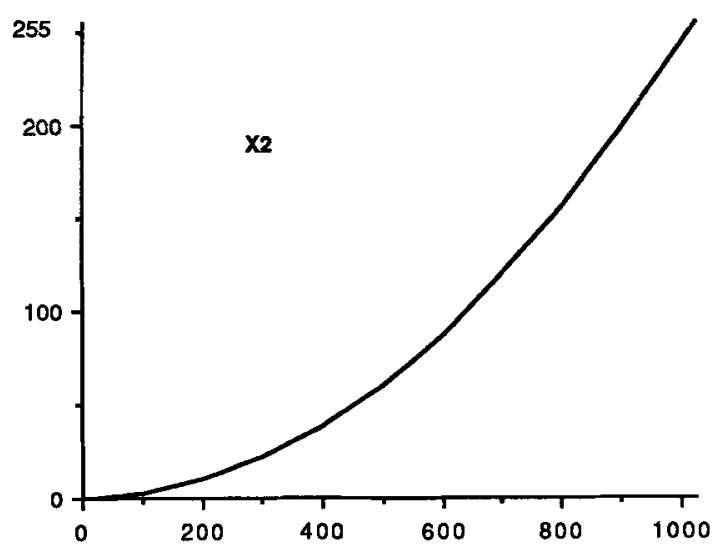

Fig 2. Square transformation.

scale and chose as a break point the mean gray-level value (Fig 4). This preferentially enhances the lung field. A transformation of type 5 can be chosen to operate on the middle range of gray-levels and leaves the low and high ends contracted, which is implemented by choosing the mean graylevel value as a break point and concatening a square function and a square root function about this breakpoint (Fig 5). Finally, transformation 6 theoretically equalizes the contrast throughout the image.

Two series of tests were conducted, the first to determine the best baseline image and second to compare this baseline image with the original analog film. In the first series, six possible baseline images are proposed for each image. These tests were run in a paired-comparison task, ${ }^{16}$ leading gradually to the final selection. Twenty-one physicians participated in the test: six emergentologists and fifteen radiologists. This last group included both board-certified radiologists as well as resident radiologists. For the second series of tests, the best baseline images are compared with the corresponding analog films by using a scale varying from -1 to +1 , where -1 is

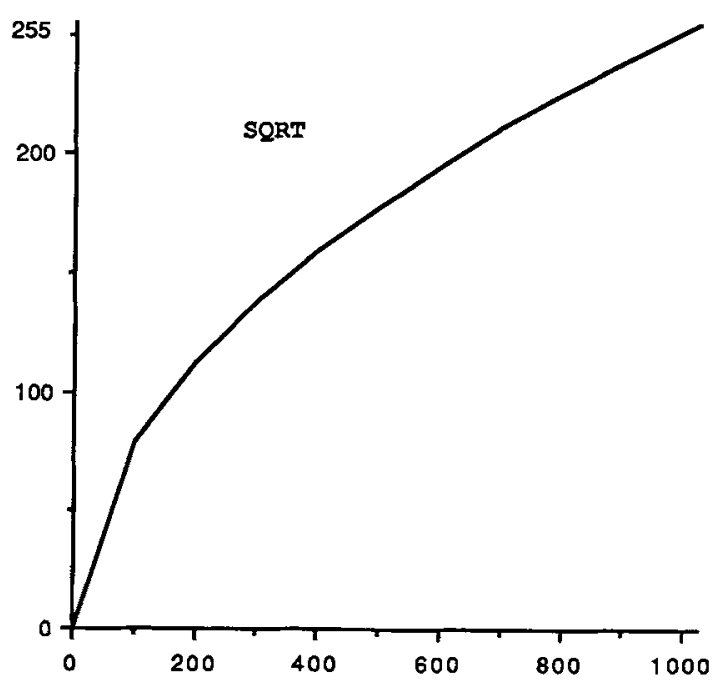

Fig 3. Square root transformation. 


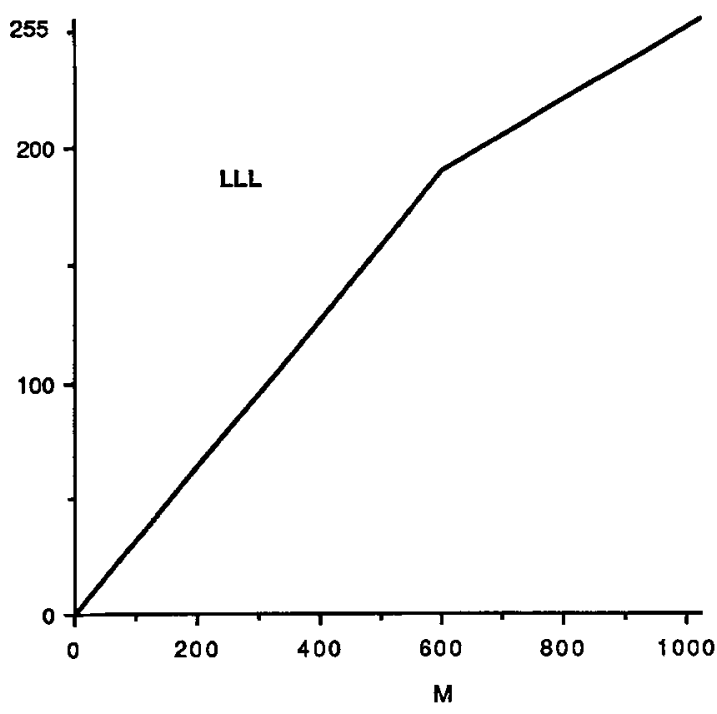

Fig 4. Piecewise linear transformation.

less clear, 0 is the same, and +1 is more clear. This latter series involved six radiologists and four emergentologists.

\section{Experiment 2: Enhancements for Local Features Analysis}

The objective of this experiment is to judge the effectiveness of simple global adaptive enhancements for the second phase in viewing a radiograph, ie, local features analysis. Attention is focused on the lungs, heart, and spine. We note that as features of interest are quite large, global adaptive enhancements are used. The questions we want to answer are as follows: How are the visibilities of the lung field, heart, and spine rated in the baseline images and in the global adaptive enhanced images? How do the enhanced digitized images compare with the analog films in terms of image-analysis effectiveness?

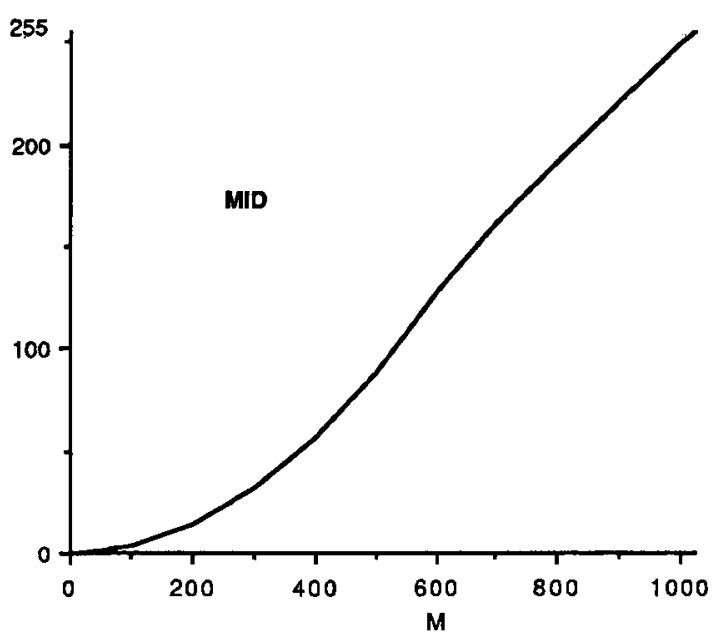

Fig 5. Middle-range stretching.
McAdams et al ${ }^{4}$ have proposed piecewise linear transformations to stretch the low and high ranges. We select one such transformation to improve the visibility in the lungs and another for the mediastinum (spine and heart areas):

Low-range stretching is used for the lungs:

$T(x)=$

$$
\left\{\begin{array}{l}
\operatorname{Int}((190 / M), x), x \in[0, M] \\
\operatorname{Int}((65 /(255-M)) \cdot(x-M)+190), x \in[M, 255]
\end{array}\right.
$$

High-range stretching is used for the mediastinum:

$\mathrm{T}(\mathrm{x})=$

$$
\left\{\begin{array}{l}
\operatorname{Int}((65 / M), x), x \in[0, M] \\
\operatorname{Int}((190 /(255-M)) \cdot(x-M)+65), x \in[M, 255]
\end{array}\right.
$$

In both these transformations, $x$ is a gray- level value, Int is the integral par, and $M$ is a breakpoint that is set to the mean of the gray level values corresponding to the thoracic cavity of the chest. These 8-bit-to-8-bit remappings are performed by simply changing the look-up table values of the display.

The six best baseline images chosen in Experiment 1 are used as the test set. The physicians rate the clarity of information for the baseline image and for the images processed by the high-stretch and the low-stretch transformations. A ranking on a scale from 1 to 5 is used, where 1 is not clear and 5 is very clear. A comparison session follows in which the best baseline image and the two processed images are compared with the analog film. A ranking on a scale from -3 to +3 is used, where -3 is much less clear, 0 is the same, and +3 is much more clear. The tests involved ten physicians: six radiologists and four emergentologists.

\section{Experiment 3: Reverse Mode Transformations}

The objective of the third experiment is to evaluate the usefulness of viewing radiographs in the reverse mode. The questions to be answered are: Is a reverse mode facility useful? What is the best image in the reverse mode?

The transformations of the first experiment are reversed (Fig 6). The black areas were transformed into white ones and the white areas into black ones without changing the contrast. The same test procedure, as used in the first experiment, is then applied. Eight radiologists participated in these tests.

\section{RESULTS}

\section{Experiment 1: Optimization of the First Overview Image}

The results of the first tests are listed in Table 1 and show the transformation rated by each physician as best for each image.

What is the enhancement that yields the best baseline image? Radiology Department images: For the first image (RAD1), the mean-range stretching (MID) is the preferred transformation in $75 \%$ of the cases. For the second image 
$g 1(x)$
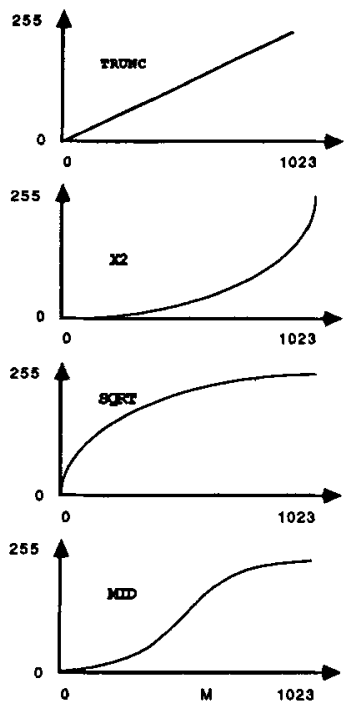

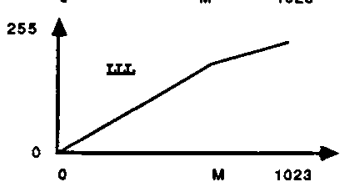

$92(x)=255-91(x)$
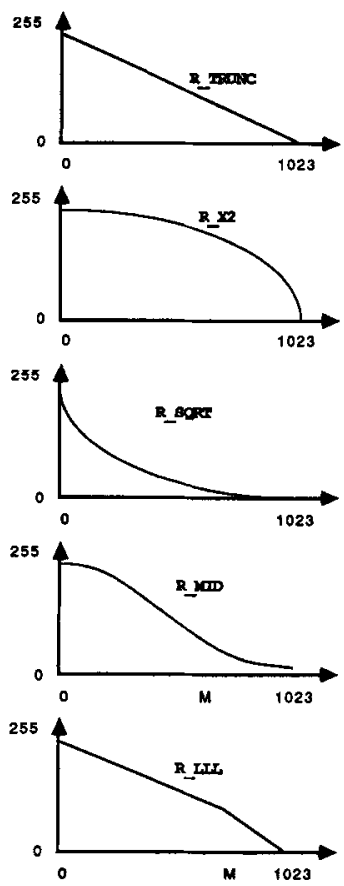

Fig 6. Reverse mode transformations.

(RAD2), the two preferred transformations are the square function (X2) and the MID.

We note that for these two images, the break point that corresponds to the mean value is fairly high $(767,721)$. The two preferred remappings are therefore very similar. In effect, the corresponding analog films presented good contrast both through the lung field and the mediastinum area. The two remappings provided digital baseline images close to the analog film. In particular, the lung field showed up clearly.

Emergency Department images: For the first image (EM1), the two preferred transformations are the square transformation (X2) and the MID. For the second image (EM2), the histogram equalization (EQU) and the square function (X2) are preferred. This image shows an overspread disease through the lung field that causes it to appear as white and washed-out. The main effect of the histogram equalization is to accentuate the contrast through the lung field.

ICU images: For the first image (ICU1), preferences are for the MID in $42 \%$ of the cases and for simple truncation (TRUNC) in $38 \%$ of the cases. For the second image, half of the physicians selected the piecewise linear transformation (LLL) that stretches the contrast through the lung field. We note that in this case the original image has a centered and fairly symmetric gray-level distribution (Fig 7).

These results, analyzed image by image and supported by the physicians' comments, show that the selection of preferred images is led by a

Table 1. Results of Experiment 1 for the Baseline Image

\begin{tabular}{cllllll}
\hline & RAD1 & RAD2 & EM1 & EM2 & ICU1 & ICU2 \\
\hline Radiologists & LLL & TRUNC & TRUNC & TRUNC & TRUNC & LLL \\
1 & MID & X2 & MID & TRUNC & EOU & LLL \\
2 & MID & X2 & X2 & EQU & MID & TRUNC \\
3 & MID & MID & X2 & EQU & MID & LLL \\
4 & MID & MID & X2 & MID & EQU & LLL \\
5 & MID & X2 & EQU & X2 & X2 & MID \\
6 & MID & MID & MID & EQU & TRUNC & LLL \\
7 & MID & MID & X2 & X2 & TRUNC & TRUNC \\
8 & X2 & X2 & X2 & X2 & MID & SQRT \\
9 & MID & MID & X2 & EQU & TRUNC & SORT \\
10 & MID & MID & X2 & TRUNC & TRUNC & SQRT \\
11 & MID & X2 & MID & X2 & TRUNC & LLL \\
12 & MID & MID & X2 & X2 & MID & TRUNC \\
13 & X2 & X2 & MID & TRUNC & TRUNC & LLL \\
14 & MID & X2 & MID & X2 & MID & LLL \\
15 & & & & & TRUNC \\
Emergentologists & X2 & MID & MID & TRUNC & LLL \\
1 & MID & MID & X2 & EOU & MID & LLL \\
2 & X2 & X2 & X2 & EOU & EQU & TRUNC \\
3 & MID & X2 & MID & MID & MID & LLL \\
4 & MID & X2 & TRUNC & TRUNC & MID & TRUNC \\
5 & MID & X2 & X2 & EOU & MID & TRUNC \\
6 & & & & &
\end{tabular}


ICU2

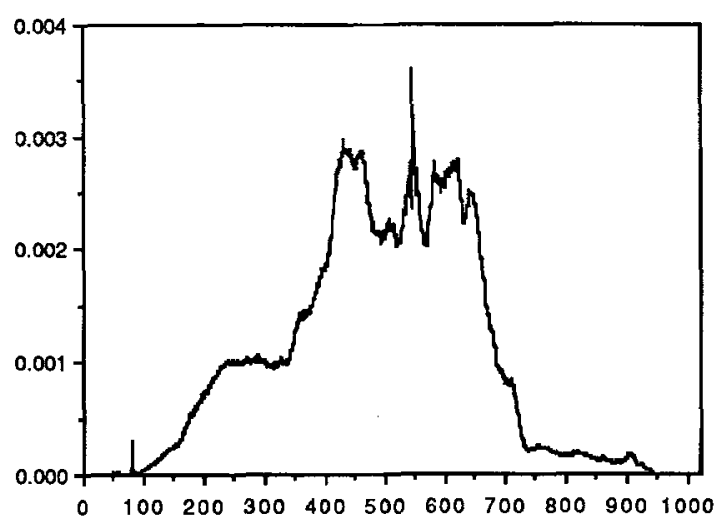

Fig 7. Image ICU2 gray level distribution.

common priority: getting satisfactory visibility in the lung field. Therefore, the baseline image judged as giving the best global impression is an image that preferably enhances the lung field.

Do the preferences vary from radiologists to emergentologists? Table 1 shows clearly that the choices of radiologists and emergentologists are highly correlated. Both the radiologists and the emergentologists chose images where the best advantage was given to the lungs.

Do the preferences depend on the radiograph acquisition technique? Definitely yes, but they also appear to correlate with image quality. In effect, Table 1 shows that the preferred transformations depend on film acquisition. However, when images are of good quality such as RAD1, RAD2, and EM1, there seems to be a clear trend for the transformations X2 and MID. For the other images, the trend is more difficult to deduce.

How do the preferred baseline images compare with the analog films? Table 2 shows how the digitized enhanced image is evaluated in comparison with the analog film. The scale varies from -1 to +1 where -1 is less clear, 0 is the same, and +1 is more clear. In general, the analog film is preferred. However, in some cases the baseline image is rated by the physicians to be as good as or better than the analog film. Experiment 2: Enhancements for Local
Features Analysis

How are the visibilities of the lungs, heart, and spine rated in the baseline image and in the global adaptive enhanced images? The results are listed in Table 3 in terms of mean and SD of rankings (between 1 and 5) for each image, each transformation, and the three features of interest: lungs, heart, and spine.

For the lungs, the baseline images yield the best visibility. The heart is also more visible in the baseline images. For the spine, high-range stretching yields the preferred images.

All the physicians agree on the good visibility of the lung field in the baseline images. However, better visibility is needed on some images for the bases and periphery of the lungs. Image EM2 showed a general pathology in the lung field, which explains its mediocre score. The low-range stretching does not improve the general visibility of the lung field. Although the lung markings are enhanced, the physicians think they are missing some information at the periphery of the lungs. The baseline image is rated as a good image for the heart. A general trend in the comments is to ask for more visibility through the heart and on its borders to bring out the margins. The heart image is not improved by low-range stretching. High-range stretching accentuates the contrast, but tends to make the heart's borders vanish. The visibility of the spine is poor in the baseline image. The radiologists indicated the need to see more clearly the pedicles, the spinus process, and the disk spaces. High-range stretching enhances the spine and these inner features are clearly brought out. Therefore, the baseline images are appropriate for the lung field and for the heart. Low-range and high-range stretching, respectively, enhance the inner part of the lungs and heart. High-range stretching clarifies the spine.

How do the enhanced digitized images compare with the analog film in terms of image analysis effectiveness? The results of the comparison session are listed in Table 4 in terms of

Table 2. Digitized Enhanced Image Compared With the Analog Film

\begin{tabular}{|c|c|c|c|c|c|c|c|c|c|c|c|c|}
\hline & \multicolumn{2}{|c|}{ RAD1 } & \multicolumn{2}{|c|}{ RAD2 } & \multicolumn{2}{|c|}{ EM1 } & \multicolumn{2}{|c|}{ EM2 } & \multicolumn{2}{|c|}{ ICU1 } & \multicolumn{2}{|c|}{ ICU2 } \\
\hline & Mean & SD & Mean & SD & Mean & SD & Mean & SD & Mesn & $\overline{\text { SD }}$ & Mean & $\overrightarrow{S D}$ \\
\hline Baseline $v$ film & -0.62 & 0.48 & -1 & 0 & -0.16 & 0.83 & -1 & 0 & -1 & 0 & -0.33 & 0.66 \\
\hline
\end{tabular}


Table 3. Persistent Experiments for the Enhanced Images

\begin{tabular}{|c|c|c|c|c|c|c|c|c|c|c|c|c|}
\hline & \multicolumn{2}{|c|}{ RAD 1} & \multicolumn{2}{|c|}{ RAD2 } & \multicolumn{2}{|c|}{ EM1 } & \multicolumn{2}{|c|}{ EM2 } & \multicolumn{2}{|c|}{ ICU1 } & \multicolumn{2}{|c|}{ ICU2 } \\
\hline & Mean & SD & Mean & SD & Mean & SD & Mean & SD & Mean & SD & Mean & SD \\
\hline \multicolumn{13}{|l|}{ Baseline } \\
\hline Lungs & 4.42 & 0.56 & 5 & 0 & 4 & 0.81 & 3 & 0.81 & 4.2 & 0.4 & 4 & 0.57 \\
\hline Heart & 4.87 & 0 & 4.5 & 0.7 & 4.16 & 0.72 & 3 & 0 & 3.8 & 0.4 & 4 & 0.57 \\
\hline Spine & 2 & 0.53 & 3 & 0 & 2.5 & 0.76 & 3.33 & 0.05 & 3.8 & 0.74 & 3.33 & 0.75 \\
\hline \multicolumn{13}{|c|}{ Low-stretch } \\
\hline Lungs & 4 & 0.75 & 4.5 & 0.7 & 3 & 1 & 1.66 & 0.05 & 4 & 0 & 3.83 & 0.4 \\
\hline Heart & 4.14 & 1 & 4 & 0 & 3.5 & 0.76 & 1.66 & 0.05 & 3.4 & 0.8 & 3.33 & 0.75 \\
\hline Spine & 1.71 & 0.88 & 2 & 0 & 1.83 & 0.69 & 1.66 & 0.05 & 2.6 & 0.8 & 2.33 & 0.47 \\
\hline \multicolumn{13}{|c|}{ High-stretch } \\
\hline Lungs & 2.29 & 0.68 & 3 & 1 & 2.33 & 0.48 & 2.33 & 0.47 & 1.2 & 0.4 & 2.5 & 0.95 \\
\hline Heart & 3.57 & 0.73 & 4 & 1 & 4 & 1.15 & 3.66 & 0.5 & 4 & 0.63 & 3.66 & 0.51 \\
\hline Spine & 3.57 & 0.5 & 4.5 & 0.7 & 3.66 & 0.77 & 4 & 0 & 4.4 & 0.49 & 4.33 & 0.5 \\
\hline
\end{tabular}

mean and SD of selected rankings (between -3 and +3 ) for each image, each transformation, and each of the three features: lungs, heart, and spine, when compared with the film.

The visibility of the lungs is slightly less clear in the baseline image than in the analog film. The heart is seen as slightly less clear to slightly more clear in the baseline image. The spine is often judged as being slightly less clear to less clear in the baseline image than in the analog film. Low-range stretching does not really improve these results for the lungs or heart and definitely degrades them for the spine. However, compared with the film, high-range stretching sometimes improves the visibility of the heart and often the visibility of the spine. These comparisons confirm the observation that the baseline image is a fairly "good" first overview and is in general close to the analog film. In some cases, the enhanced images also improve the visibility of features as compared with the analog film.

\section{Experiment 3: Reverse Mode Transformation}

The results of this experiment are listed in Table 5. They show the reversed transformation rated by each physician as "best" for each image.

Is a reverse mode transformation useful? All the physicians agreed that viewing images in the reverse mode could improve the visibility of certain areas and the detection of certain features.

What is the best image to be displayed in the reverse mode? Table 5 shows that there is a high degree of concurrence in the radiologists' preferences regarding the aspect of a baseline image in the reverse mode. However, these baseline images in the positive mode do not correspond to reversing the negative selected baseline images. The preferred images in the reverse mode are the reversed truncation (R TRUNC) in $54 \%$ of the cases and the reversed piecewise linear function (R LLL) in $29 \%$ of the cases. Contrary to experiment 1 , the results are almost

Table 4. Results of Experiment 2 Comparing the Enhanced Images With the Film

\begin{tabular}{|c|c|c|c|c|c|c|c|c|c|c|c|c|}
\hline & \multicolumn{2}{|c|}{ RAD1 } & \multicolumn{2}{|c|}{ RAD2 } & \multicolumn{2}{|c|}{ EM1 } & \multicolumn{2}{|c|}{ EM2 } & \multicolumn{2}{|c|}{ ICU1 } & \multicolumn{2}{|c|}{ ICU2 } \\
\hline & Mean & SD & Mean & So & Mean & So & Mean & SD & Mean & SD & Mean & SD \\
\hline \multicolumn{13}{|l|}{ Baseline } \\
\hline Lungs & -0.87 & 0.36 & -0.5 & 0.25 & -0.66 & 0.89 & -1.66 & 0.24 & -0.6 & 0.24 & -0.83 & 0.14 \\
\hline Heart & 0.12 & 0.33 & -0.5 & 0.25 & 0.16 & 0.13 & 0 & 0 & -0.2 & 0.96 & -0.16 & 0.13 \\
\hline Spine & -1.12 & 0.6 & 0 & 1 & -0.83 & 0.47 & 0.33 & 0.55 & -1.2 & 0.16 & -1 & 0 \\
\hline \multicolumn{13}{|c|}{ Low-stretch } \\
\hline Lungs & -0.5 & 1.25 & -1.5 & 0.25 & -1.5 & 0.91 & -1.66 & 0.24 & -1.2 & 0.56 & -0.33 & 1.55 \\
\hline Heart & -0.62 & 0.74 & -1 & 1 & -1 & 0.33 & -1.33 & 0.23 & -0.8 & 1.36 & 0 & 0.66 \\
\hline Spine & -2.12 & 0.85 & -2 & 0 & -2 & 1 & -1.33 & 0.89 & -1.8 & 2.16 & -2.66 & 0.25 \\
\hline \multicolumn{13}{|c|}{ High-stretch } \\
\hline Lungs & -2.12 & 0.38 & -2.5 & 0.25 & -2.83 & 0.15 & -2 & 0.66 & -2.8 & 0.16 & -2 & 0.5 \\
\hline Heart & -1 & 0.5 & 0 & 1 & 0.33 & 0.22 & 0.66 & 0.22 & -0.4 & 0.64 & 0 & 1.33 \\
\hline Spine & 0.62 & 0.24 & 0 & 0 & 0.83 & 0.97 & 1 & 0 & 0 & 0 & 1 & 0.33 \\
\hline
\end{tabular}


Table 5. Results of Experiment 3

\begin{tabular}{|c|c|c|c|c|c|c|}
\hline Radiologists & RAD 1 & RAD2 & EM1 & EM2 & ICU1 & ICU2 \\
\hline 1 & R TRUNC & R TRUNC & R TRUNC & R SORT & R LLL & R TRUNC \\
\hline 2 & R LLL & R TRUNC & R TRUNC & R SORT & R LLL & R TRUNC \\
\hline 3 & R LLL & $\mathrm{R} \times 2$ & R MID & R LLL & R TRUNC & R MID \\
\hline 4 & R TRUNC & R TRUNC & R LLL & R SORT & R TRUNC & R TRUNC \\
\hline 5 & R TRUNC & R TRUNC & R TRUNC & R LLL & R LLL & R TRUNC \\
\hline 6 & R LLL & R TRUNC & R TRUNC & R SORT & R LLL & R LLL \\
\hline 7 & R LLL & R TRUNC & R TRUNC & R LLL & R TRUNC & R TRUNC \\
\hline 8 & R TRUNC & $\mathrm{R} \times 2$ & R TRUNC & R LLL & R TRUNC & R TRUNC \\
\hline
\end{tabular}

similar from one image to another and correspond either to leave the image unchanged or to stretch the low range of gray levels. The smaller disparities between preferences for each image may be attributed to the unusual aspect of reverse mode images and the physicians' lack of practice in discriminating features in these images. The best images in the reverse mode do not generally correspond to simply reversing the best baseline images in the usual positive mode.

\section{CONCLUSION}

Our approach to context-dependent enhancements attempts to match the three phases involved in viewing a radiograph: getting a global impression, analyzing the objects and the local features, and focusing on image perturbations. In this article, we reported on enhancements to support the two first phases in the case of chest radiographs. In addition, results on the applicability of gray-level reversal transformations have also been reported.

The baseline view judged as giving the best global impression is an image that first provides satisfactory visibility in the lung field. However, enhancements that yield the best baseline images are difficult to deduce when the original images are of poor quality. If the original images are of good quality, the consensus appears to be enhancements in the high range or mean range.
Global adaptive gray-scale manipulations yield a global change that is the result of an enhancement focused on a part of the radiograph. They are useful as secondary images to support the analysis of the objects and of the local features in images, in particular those of the spine, the inside of the lungs and heart. In terms of visibility of the lungs, heart, and spine, we get results close to those of the analog film in using one transformation or the other, including the one that yields the baseline image, which would suggest an implementation with preset enhancements for these areas under the user control.

The last experiment asserts the usefulness of viewing radiographs in the reverse mode. However, a good image in the reverse mode does not necessarily correspond to the reversal of the baseline image in the usual mode. In general, the preferred images correspond to reversing the simple truncation function. Implementing a reverse mode facility requires the knowledge of the appropriate 8-bit-to-8-bit remapping that transforms the baseline image in the usual mode to the desired one in the reverse mode.

\section{ACKNOWLEDGMENT}

The authors wish to thank the radiologists, emergentologists, and personnel of the Ottawa Civic Hospital, who generously donated time to their experiments.

\section{REFERENCES}

1. Johnson GA, Danieley N, Ravin CE: Processing alternatives for digital chest imaging. Radiol Clin North Am 23:335-340, 1985

2. Huang HK: Elements of Digital Radiology. Englewood Cliff, NJ, Prentice-Hall, 1987

3. Cocklin ML, Gourlay AR, Jackson PH, et al: Digital processing of chest radiographs. Images Vis Comput 1:67, 78, 1983

4. McAdams HP, Johnson GA, Suddarth A, et al: Histogram directed processing of digital chest images. Invest Radiol 21:253, 1986

5. Pizer SM, Amburn EP, Austin JD, et al: Adaptive histogram equalization and its variations. Comput Vision, Graphics Image Processing 39:355-368, 1987

6. Sherrier RH, Johnson GA: Regionally adaptive histogram equalization of the chest. IEEE Trans Med Imaging MI 6:1-7, 1987

7. McAdams PA, Johnson AG, Suddarth SA, et al: Implementation of adaptive filtration for digital chest imaging. Optical Engineering 26, 7:669, 672, 1987

8. Fraser RG, Breatnach E, Barnes GT: Digital radiography of the chest: Clinical experience with a prototype chest unit. Radiology 148:1-5, 1983 
9. Goodman LR, Foley WD, Wilson CR, et al: Digital and conventional chest images: Observer performance with filmdigital radiography system. Radiology 158:35-39, 1986

10. Goodman LR, Foley WD, Wilson CR, et al: Pneumothorax and other lung diseases: Effect of altered resolution and edge enhancement on diagnosis with digitized radiographs. Radiology 167:83-88, 1988

11. Oestmann JW, David CK, Bourgoin PM, et al: Subtle lung cancers: Impact of edge enhancement and gray scale reversal on detection with digitized chest radiographs. Radiology 167:657-658, 1988

12. Sherrier RH, Chiles C, Wilkinson WE, et al: Effects of image processing on nodule detection rates in digitized chest radiographs: ROC study of observer performance. Radiology 166:447-450, 1988

13. Blume $\mathrm{H}$, Kamiya $\mathrm{K}$ : Auto-ranging and normalization versus histogram modifications for automatic image processing of digital radiographs. SPIE Medical Imaging 767: 371-383, 1987

14. Nodine CF, Kundel HL: Using eye movement to study visual search and to improve tumor detection. Radiographics 7:1241-1250, 1987

15. Smathers RL, Brody WR: Digital radiography: Current and future trends. Br J Radiol 58:285-307, 1985

16. Coombs CH, Dawes RM, Tversky A: Mathematical Psychology: An Elementary Introduction. Englewood Cliffs, NJ, Prentice-Hall, 1970 